\title{
Mechanism Design for Dynamic Settings
}

\author{
RUGGIERO CAVALLO \\ University of Pennsylvania
}

\begin{abstract}
We provide a brief summary of dynamic mechanism design: what it is, what purpose it serves, and some of the main results to date. We then describe work that applies the theory to an auction setting with deliberative agents and, briefly, work that extends the theory to settings where the population of agents changes over time. We conclude with a discussion of directions for future research.

Categories and Subject Descriptors: J.4 [Computer Applications]: Social and Behavioral Sciences-Economics

General Terms: Economics, Algorithms, Theory

Additional Key Words and Phrases: Dynamic mechanism design, VCG, Social welfare
\end{abstract}

Mechanism design is the discipline of designing rules that lead to socially desirable outcomes in a context where individuals are selfish and hold private information. Agents are asked to report their private information to a social planner, who selects an outcome and then makes payments carefully defined so that each individual maximizes his utility by reporting truthfully. Mechanism design has historically focused almost exclusively on a static model, either where there is just a single decision to be made ("one-shot" settings) or where all private information that will be relevant to any future decisions is known to participants at the beginning of the mechanism.

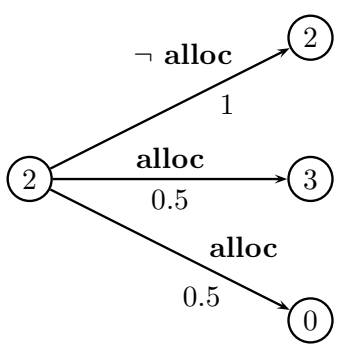

Fig. 1. A concise representation of dynamic type for one agent in a 2 time period allocation decision. In the first period the agent's value for receiving the item is 2 ; if not allocated then, the second period value is still 2 , otherwise it is either 0 or 3 with equal probability.
But of course many settings are not, in fact, static in this way. Future decisions may depend on the (non-deterministic) outcomes of past decisions. Consider repeated allocation of a single good, allocated for one week intervals in order to maximize total social utility over the period of a month. After the first allocation decision the agent who obtained the good may learn something about how he values it; hence he will have obtained new private information, knowledge of which is crucial for making optimal subsequent decisions. Agent types are inherently dynamic here, and an appropriate representation is a Markov decision process (MDP) rather than a single value (see Figure 1).

In dynamic settings the solutions of static mechanism design are insufficient.

Author's email address: cavallo@seas.upenn.edu 
For instance, running the static VCG mechanism every period, ignoring the type dynamics, will not yield truthfulness as an equilibrium strategy. Over the past few years a series of papers has started to address this problem of dynamic mechanism design. In a dynamic mechanism, agents are asked to report private information and payments are potentially made every time period. To give a slightly overbroad yet reasonable summary, we can say that several of the central results of efficient (i.e., social-welfare maximizing) static mechanism design have been shown to have analogs in the dynamic setting that are very close in spirit, if significantly more complex.

\section{SOME CORE RESULTS}

In static mechanism design the central positive result is that the Groves class of mechanisms, in which efficient outcomes according to agent reports are chosen and each agent is payed the reported welfare of the other agents minus some quantity independent of their report, characterizes the space of mechanisms that yield efficient outcomes in dominant strategies [Green and Laffont 1977; Holmstrom 1979]. In [Cavallo et al. 2006] and [Athey and Segal 2007] it is shown that the most simple and natural dynamic extension of this, in which each period each agent is payed the value reportedly achieved by other agents, is truthful in within-period ex post Nash equilibrium. Intuitively, this equilibrium requires that each agent achieve the maximum possible value going forward, whatever the types of the other agents, on the condition that other agents play the equilibrium going forward. ${ }^{1}$ In [Cavallo 2008 ] it is shown that the dynamic-Groves class of mechanisms - defining payments such that each agent's expected cumulative utility forward equals social welfare minus a quantity independent of the agent's reporting strategy - characterizes the entire space of dynamic mechanisms that are efficient in within-period ex post Nash equilibrium while meeting an intuitive history-independence condition.

Within the space of within-period ex post efficient mechanisms, there are superlative members with regard to budgetary or individual rationality properties. In an early paper, Cavallo, Parkes, and Singh [2006] provide a mechanism which, from the beginning of execution, is weakly budget-balanced in expectation and in which no agent expects to obtain negative utility from participating. ${ }^{2}$ In an important advance, Bergemann and Välimäki [2006] strengthen these properties, obtaining a mechanism that is individual rational and no-deficit in expectation every period, regardless of the sequence of state transitions that is realized. Their dynamic-VCG mechanism captures the spirit of the static VCG mechanism in that each agent's expected utility forward from any state equals his expected cumulative (over time) marginal contribution to social welfare. Intuitively, in each time-step dynamicVCG charges each agent an amount equal to the expected extent to which that agent's current type report prevents other agents from obtaining utility now and

\footnotetext{
${ }^{1}$ Dominant strategy equilibrium is less meaningful in dynamic, non-deterministic settings because it requires that an agent cannot benefit regardless of how outcomes are (randomly) realized. This would require, for instance, that an agent never regret obtaining a good if in later periods his value for it unexpectedly decreased.

${ }^{2}$ So that agents would be willing to sign a contract obligating them up-front to participation in the mechanism, but may wish they could drop out if things go poorly.
}

ACM SIGecom Exchanges, Vol. 8, No. 2, December 2009 
in the future. Dynamic-VCG is a member of the dynamic-Groves class, so it is within-period ex post efficient.

In [Cavallo 2008] it is shown that among all within-period ex post efficient and individual rational mechanisms that meet a history-independence condition, dynamicVCG is revenue maximizing: no other such mechanism ever yields more expected revenue on any problem instance, from any joint state that might arise. In auction settings this fact makes dynamic-VCG desirable, at least from the perspective of the auctioneer. However, if the goal is truly to optimize social-welfare, i.e., maximize the utility of the agents, then in a sense dynamic-VCG is the worst efficient mechanism; the ideal mechanism would be strongly budget-balanced, with the agents maintaining all the surplus while payments merely move money around among them. For environments that can be modeled as multi-armed bandits, ${ }^{3}$ in [Cavallo 2008] a dynamic redistribution mechanism is provided that comes very close to strong budget-balance without sacrificing the desirable within-period ex post efficiency, individual rationality, or no-deficit properties of dynamic-VCG. ${ }^{4}$ For agent types distributed uniformly or normally, for population size greater than four agents, the mechanism maintains over $90 \%$ of the value within the group of agents whereas dynamic-VCG maintains less than $20 \%$.

Athey and Segal [2007] provide a dynamic mechanism that does even better in terms of budget-balance and is applicable to general settings. ${ }^{5}$ Their dynamicbalanced mechanism is strongly budget-balanced, although it is efficient only in the weaker Bayes-Nash equilibrium and fails to satisfy within-period ex post individual rationality. Like the mechanism of [Cavallo et al. 2006], it is ex ante individual rational: agents would "sign up" for the mechanism if given the choice at the beginning.

\section{AN APPLICATION: AUCTIONS WITH DELIBERATIVE AGENTS}

The extension dynamic mechanism design makes to previous theory is important because so many decision settings have a crucial non-static element, even settings that do not seem overtly dynamic at first glance. One example is that studied in [Cavallo and Parkes 2008], in which a single resource is to be allocated once, but where the agents potentially interested in the item have values for it that can be modified by research or "deliberation". ${ }^{6}$ One can imagine companies competing to obtain a new technology that is at first poorly understood; a company may have an idea for how to use the technology in a way that yields value $x$, but further research - which could come at a cost - may (non-deterministically) uncover a business plan under which the technology yields value $y>x$. Agent types here can be represented as MDPs as in Figure 2. A social planner can optimize social welfare by directing the sequence of deliberation actions to be taken by the agents prior to an allocation decision.

\footnotetext{
${ }^{3}$ E.g., most saliently, settings of repeated allocation of a single item.

${ }^{4}$ The mechanism is, in spirit, an extension of the redistribution mechanism of [Cavallo 2006] for static environments.

${ }^{5}$ All the work discussed here makes an assumption akin to private values, though correlated types are allowed.

${ }^{6}$ One of the first papers considering mechanism design for deliberative agents is [Larson and Sandholm 2005].
} 


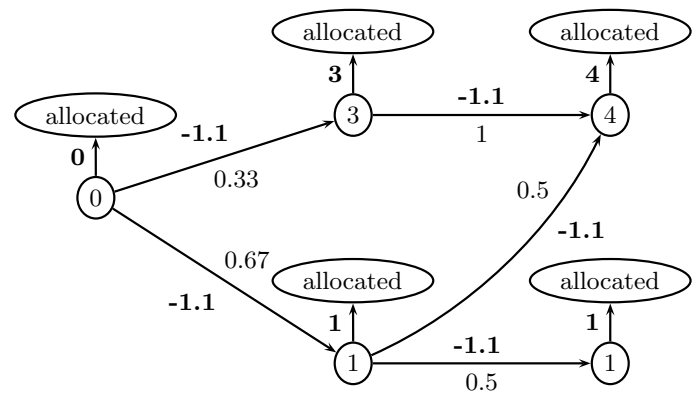

Fig. 2. Representation of a deliberative agent's type, with deliberation costs (1.1) and transition probabilities labeled on arcs, and nodes labeled with value for allocation. A maximum of two deliberation steps are potentially useful. Allocation decisions are represented in the vertical arcs, and deliberation actions in the rightward-progressing arcs.

Even in this setting where a one-time allocation is made, a dynamic mechanism is required in order to bring the efficient sequence of deliberations and ultimate allocation into equilibrium. Cavallo and Parkes [2008] show that an application of dynamic-VCG is a solution here when agents can deliberate only about their own values, and moreover that the mechanism can be modified to handle cases where agents can strategically deliberate about other agents' values. It is also shown that computing the optimal policy can be reduced to a multi-armed bandits problem and is thus computationally tractable.

\section{DYNAMIC POPULATIONS}

Preceding the recent work on dynamic mechanism design is so-called "online mechanism design", where agents arrive and depart (the population changes) but types are static (agents obtain no new private information after arrival). ${ }^{7}$ In recent work, Cavallo et al. [2009] unify these two strands of research, adapting the dynamicGroves class of mechanisms in general and dynamic-VCG in particular to handle the problem of maximizing social welfare when agents with dynamic type arrive and depart. In such settings a successful mechanism must provide incentives for agents to truthfully report types, and also to be truthful in announcing their true arrival rather than "hiding" from the mechanism.

\section{SOME FUTURE DIRECTIONS}

Dynamic mechanism design is a young subfield and there are many important directions still to be pursued. Perhaps the most glaring issue in this whole line of work - the "elephant in the room"-is computation: we have positive results in the form of mechanisms with very desirable equilibrium properties, but these desirable properties hold on the condition that the social planner executes a socially optimal decision policy and, unfortunately, computing such a policy is frequently intractable. Is it interesting to talk about solutions that can't be implemented in practice? One way of approaching this issue is to move to less stringent solution concepts; another is to focus on settings that are structured in a way that admits

\footnotetext{
${ }^{7}$ See [Parkes 2007] for a nice survey. 
tractable optimal solutions; [Cavallo and Parkes 2008] is one such example, with its reduction of the problem to a multi-armed bandits setting.

Another open direction is dynamic mechanism design for settings with interdependent values. The dynamic-VCG mechanism and others assume that the value an agent obtains in any particular period, given the decision made by the center, depends only on his own private state (type) and not that of the other agents. It is far from apparent that the dynamic-VCG mechanism can be extended to interdependent settings, and so the important question of designing a within-period ex post efficient, individual rational, and no-deficit mechanism there is currently open. $^{8}$

\section{REFERENCES}

Athey, S. And Segal, I. 2007. An efficient dynamic mechanism. Working paper, http://www.stanford.edu/ isegal/agv.pdf.

Bergemann, D. And Valimaki, J. 2006. Efficient dynamic auctions. Cowles Foundation Discussion Paper 1584.

Cavallo, R. 2006. Optimal decision-making with minimal waste: Strategyproof redistribution of VCG payments. In Proc. 5th Int. Conf. on Autonomous Agents and Multi-Agent Systems (AAMAS'06).

Cavallo, R. 2008. Efficiency and redistribution in dynamic mechanism design. In Proceedings of the 9th ACM Conference on Electronic Commerce (EC-08).

Cavallo, R. and Parkes, D. C. 2008. Efficient metadeliberation auctions. In Proceedings of the 26th Annual Conference on Artificial Intelligence (AAAI-08).

Cavallo, R., Parkes, D. C., And Singh, S. 2006. Optimal coordinated planning amongst selfinterested agents with private state. In Proceedings of the Twenty-second Annual Conference on Uncertainty in Artificial Intelligence (UAI'06).

Cavallo, R., Parkes, D. C., And Singh, S. 2009. Efficient mechanisms with dynamic populations and dynamic types. In submission.

Green, J. And LAFFont, J.-J. 1977. Characterization of satisfactory mechanisms for the revelation of preferences for public goods. Econometrica 45, 427-438.

Holmstrom, B. 1979. Groves' scheme on restricted domains. Econometrica 47(5), 1137-1144.

Larson, K. And Sandholm, T. 2005. Mechanism design and deliberative agents. In Proceedings of the Fourth International Joint Conference on Autonomous Agents and Multiagent Systems (AAMAS 2005).

Parkes, D. C. 2007. Online mechanisms. In Algorithmic Game Theory, N. Nisan, T. Roughgarden, E. Tardos, and V. Vazirani, Eds. CUP.

\footnotetext{
${ }^{8}$ See the final section of [Cavallo et al. 2009] for a more detailed discussion of this problem.
} 